\title{
Metal-free four-step domino reaction enables fully controlled non- statistical synthesis of hexaarylbenzene with six different aryl groups
}

${ }^{1}$ Organic Chemistry Chair I and Interdisciplinary Center for Molecular Materials (ICMM), Friedrich-AlexanderUniversität Erlangen-Nürnberg, Nikolaus-Fiebiger-Straße 10, 91058 Erlangen, Germany

${ }^{2}$ Organic Chemistry Chair II and Interdisciplinary Center for Molecular Materials (ICMM), Friedrich-AlexanderUniversität Erlangen-Nürnberg, Nikolaus-Fiebiger-Straße 10, 91058 Erlangen, Germany

${ }^{3}$ Leibniz Institute of Surface Engineering (IOM), Permoserstr. 15, 04318 Leipzig, Germany

* Corresponding author. Email: svetlana.tsogoeva@fau.de

Abstract: Hexaarylbenzene (HAB) derivatives are versatile aromatic systems playing a significant role as chromophores, liquid crystalline materials, molecular receptors, redox materials, organic light-emitting diodes and photochemical switches. Statistical synthesis of simple symmetrical HABs is known via lowyielding cyclotrimerization or Diels-Alder reactions. By contrast, the synthesis of more complex, asymmetrical systems, and without involvement of statistical steps, remains an unsolved problem. Here we present a generally applicable synthetic strategy to access asymmetrical HAB via an atom-economical and high-yielding metal-free four-step domino reaction using nitrostyrenes and $\alpha, \alpha$-dicyanoolefins as easily available starting materials. Resulting domino product - functionalized triarylbenzene (TAB) - can be used as a key starting compound to furnish asymmetrically substituted hexaarylbenzenes in high overall yield and without involvement of statistical steps. This straightforward domino process represents a distinct approach to creating diverse and still unexplored HAB scaffolds, containing six different aromatic rings around central benzene core.

Benzene, a six-membered hydrocarbon aromatic ring system, is one of the most abundant structural features in chemistry, ranging e.g. from natural products ${ }^{1}$, ligand systems for metal complexes $^{2}$ to pharmaceutics ${ }^{3}$. The benzene ring possesses manifold interesting electronic properties which makes it indispensable as building block in expanded conjugated systems used as organic semiconductors ${ }^{4-7}$. Expansion of polycyclic aromatic hydrocarbons leads to a smaller gap between HOMO and LUMO of the molecule - which means they can be more easily transferred into their excited state: as a result, these molecules absorb in the UV or visible light range, are often colored and find use as photosensitizers, dyes or chromophores ${ }^{8}$.

If every hydrogen atom in benzene is replaced by further benzene rings, the system is expanded to hexaphenylbenzene (HPB, Fig. 1a), which exhibits propeller chirality and toroidal delocalization? By substitution of the outer phenyl rings with the same or different functional groups, these derivatives can be described in general as hexaarylbenzenes (HAB, Fig. 1a). These systems are of high interest ${ }^{10}$, as they have been already established in multiple applications like molecular electronics $^{11}$, spintronics $^{12}$, chromophores $^{13}$, catalysts ${ }^{14}$ or, by Scholl-oxidation to hexabenzocoronene (HBC), which is used as model for graphene ${ }^{15}$ or its synthesis ${ }^{16}$. As a result, construction of novel asymmetrical HABs, their investigation and fine tuning of their properties, is of high demand. However, existing synthetic methods, like Diels-Alder reactions ${ }^{17}$, cyclotrimerization $^{18,19}$ or Suzuki-coupling of multi-halogenated derivatives ${ }^{20}$, do not offer full control of the final HAB constitution, i.e. access to all 36 combinatorically possible constitution isomers or substitution patterns for arbitrary substituents ${ }^{21}$. Moreover, for a given six different aryl 
groups around benzene core, there are $6 ! / 6=120$ distinguishable isomers possible (because each of the six substituents can be assigned to any of the six core benzene carbons), comprising of 60 position isomers that exist each as a pair of - rapidly racemizing - enantiomers, due to propeller chirality of the $\mathrm{HAB}^{22}$. As a result, conventional synthesis gives complex mixtures of very similar molecules, bearing similar physical properties. Separation and isolation of a desired single target HAB is hardly possible and only in low yield (Fig. 1b). Moreover, examples of controlled postmodification of HAB scaffolds are still very $\operatorname{rare}^{18}$. Hence, these standard methodologies are not feasible to obtain more complex, asymmetrically substituted systems. Under these circumstances it is not surprising that to date there is only one single example for a $\mathrm{HAB}$, bearing six differently substituted phenyl rings ${ }^{23}$. Although the approach, which functionalizes 2-methoxy thiophene, marks a great milestone, it suffers from low overall yields, small scales and harsh reaction conditions. Additionally, final [4+2] cycloaddition is a statistical reaction, which results in two different products when employing an asymmetric tolan (Fig. 1c). The mixture of stereoisomers was inseparable without post-modification of the final product, therefore critically limiting the applicability of this method. The challenge of designing a general, reliable and non-statistical route for synthesis of HABs is, therefore, not yet met, which makes development of new synthetic approaches highly desirable.

In addition to conventional "stop-and-go" synthetic methodologies, domino reactions have attracted high attention since recently. These processes include a cascade of multiple reactions which proceed sequentially and where previous step triggers its successor, yielding complex molecules with a single workup and purification process. Thereby domino processes are time saving, waste reducing, atom efficient and can be considered a green method superior to step-wise synthesis approaches ${ }^{24-26}$. Even more cost efficient are metal-free multi-step domino reactions, as these are much less expensive than those using metal catalysts. ${ }^{27-30}$.

Having this in mind, we developed a novel 4-step domino-process yielding hexa-substituted triarylbenzene (TAB) derivatives to fill the existing gap in non-statistical synthetic methods towards HAB (Fig. 1d). The domino sequence, employing easily available precursors, gives full control of the substitution pattern on the central benzene core. The three additional and different functional groups on the core-ring of TAB domino product allow stepwise introduction of three further aromatic rings via further synthetic steps. This approach offers the first scalable synthetic method towards HAB without any statistical steps, which allows access to complete theoretically possible chemical space for HABs in a pre-designed way. 


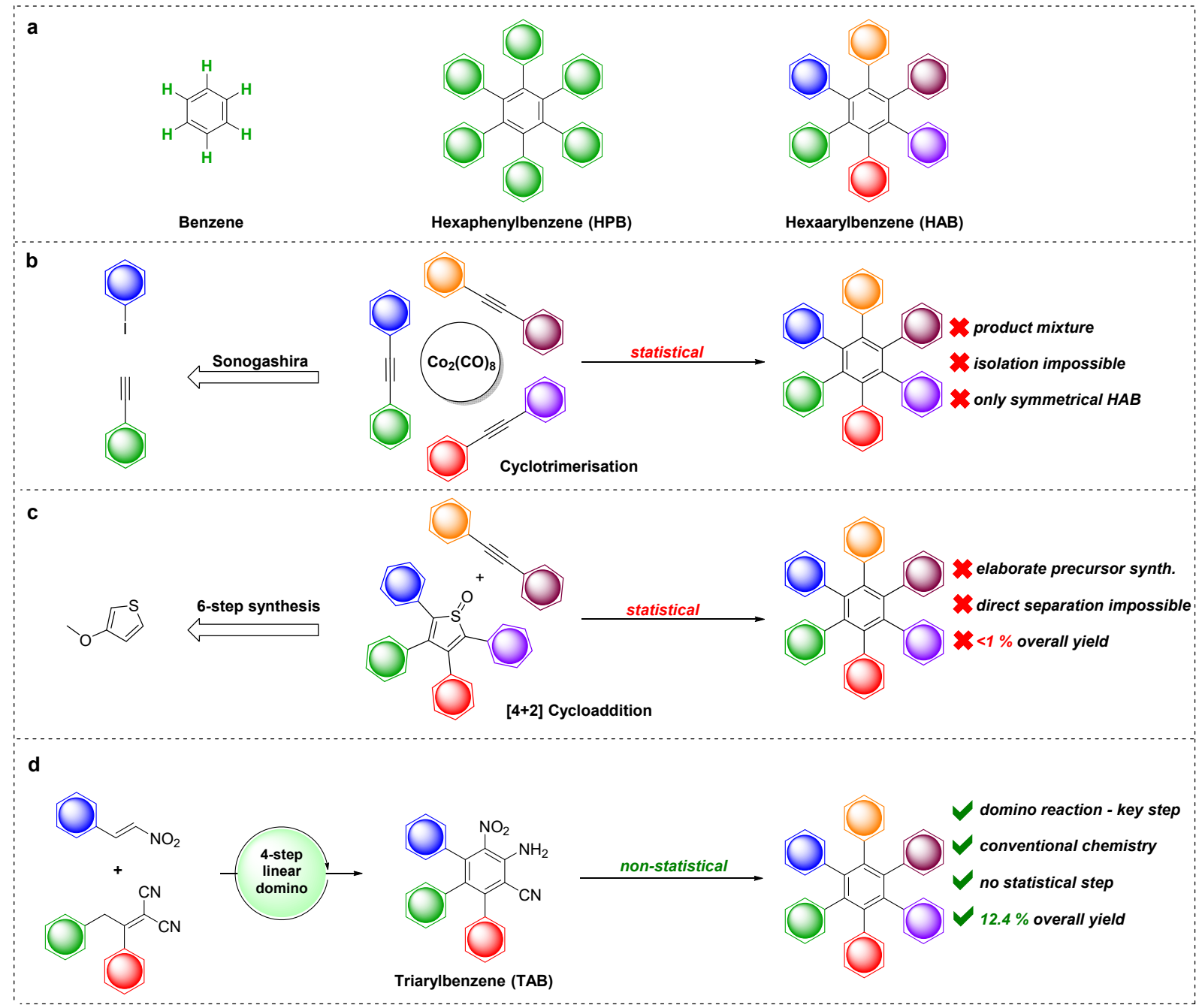

Fig. 1 | Outline of known and proposed synthesis of HABs. a, Benzene and hexaarylbenzene. b, Shortcomings of established synthetic methods like cyclotrimerization or $\mathbf{c}$, Diels-Alder reaction. $\mathbf{d}$, This work: novel approach to the synthesis of hexaarylbenzene with complete control over aryl-installing steps.

\section{A novel domino reaction as a key unlocking the non-statistical synthesis of hexaarylbenzene: Reaction development}

We set out by investigating three-component domino reaction of 4-methylnitrostyrene (1a), 1-(4fluorophenyl)-2-phenylethan-1-one and malononitrile using DABCO as catalyst and acetonitrile as solvent (Fig. 2a). However, no conversion towards desired product $6 \mathbf{a}$ was observed, since Knoevenagel condensation step was not feasible under the chosen conditions (see Fig. S1). Instead, Michael product of nitrostyrene and malononitrile (see Fig. S2) preferably forms, and reacts further to a complex mixture of undesired products (see Fig. S3 and Fig. S4). Hence, we decided to use preformed Knoevenagel product 2a in the domino reaction towards TAB 6a (Fig. 2b). 


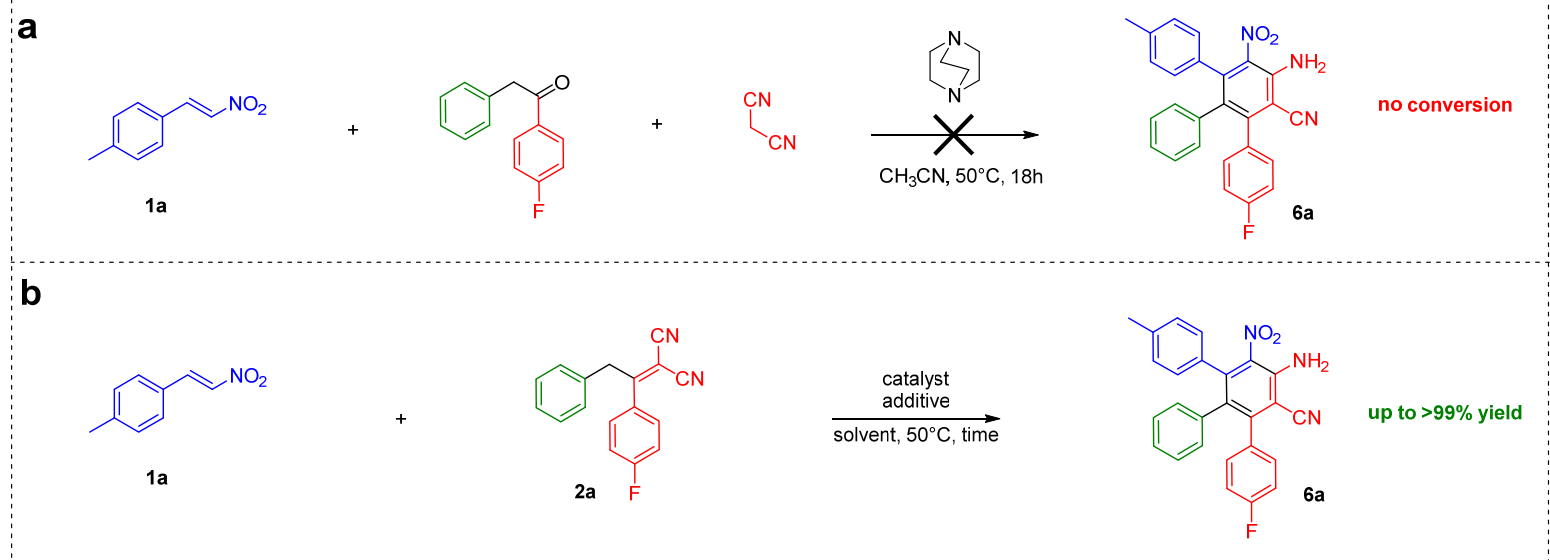

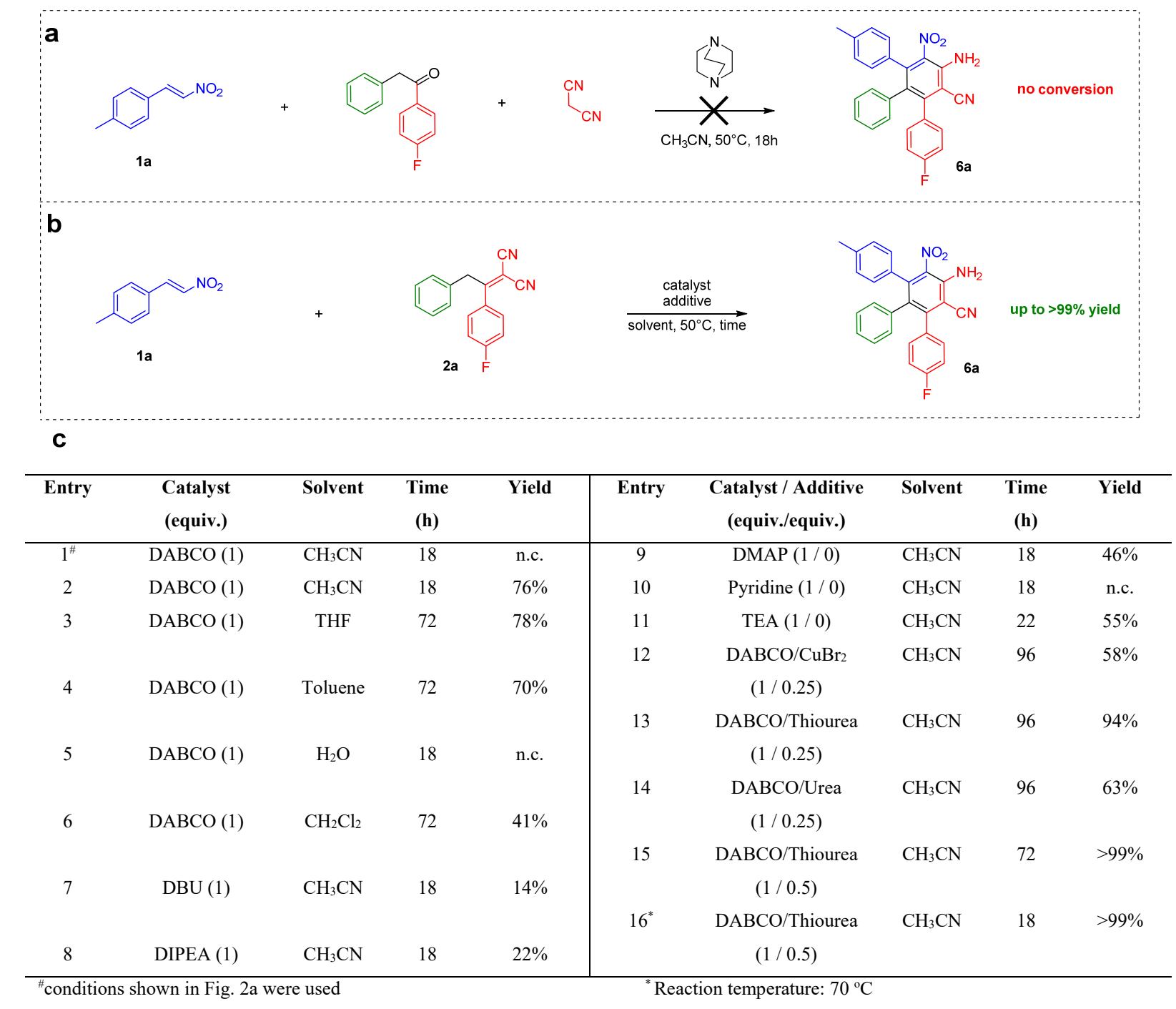

Fig. 2 | Development of novel domino reaction. a, Initial attempt of synthesizing TAB compound 6a via three-component domino reaction (see entry 1). b, Developed two-component four-step domino reaction towards TAB $6 \mathbf{6}$ (entries 2-16), additional entries can be found in Table S1. c, Screening of reaction conditions.

To our delight, this second attempt of the two-component domino reaction using DABCO in acetonitrile already resulted in good yield of $76 \%$ after $18 \mathrm{~h}$ (Fig. 2c, entry 2). As we were able to identify the cyclohexadiene intermediate 5a (Fig. 3d), we prolonged the reaction time till the corresponding spots of reactants on TLC disappeared completely. Extensive screening of solvents (Fig. 2c, entries 3-6) and catalysts (Fig. 2c, entries 7-11) did not result in any improvements, so we focused on using different additives (Fig. 2c, entries 12-16). While copper(II)bromide (Fig. 2c, entry 12) and urea (Fig. 2c, entry 14) were decreasing the yield, addition of thiourea improved the yield significantly to $94 \%$ (Fig. 2c, entry 13). After optimizing the amounts of additive (Fig. 2c, entry 15 ) and the temperature (Fig. 2c, entry 16), we were able to isolate the desired product in quantitative yield. 
Having now the optimized reaction conditions in hand, we next explored the scope of reaction to investigate effect of different types of substituents at aromatic rings of TAB (Fig. 3a). To show the power of the reaction and demonstrate its versatility, we first synthesized derivatives $6 \mathbf{b}-6 \mathbf{m}$ (Fig. $3 \mathrm{~b}$ ). By simply varying the corresponding starting materials, we were able to construct TABs with electron donating or electron withdrawing groups selectively on each of the three rings in up to $88 \%$ yield (i.e. average of $97 \%$ per step). Since no clear trends of substituents' influence on reaction outcome was observable, we additionally synthesized derivative $\mathbf{6 n}$ with unsubstituted phenyl rings. The moderate yield of $40 \%$ confirmed that substituents in general seem to have a beneficial influence on the overall yield. In addition, we also synthesized derivatives $\mathbf{6 0}$ and $\mathbf{6 p}$, which might be interesting candidates for post-modifications e.g., for synthesis of $\mathrm{CF}_{3}$-containing bioactive quinazoline-scaffolds (starting from 6o) or Suzuki-coupling reactions (using 6p). Finally, we employed sterically hindered nitrostyrenes leading to $\mathbf{6 q}, \mathbf{6 r}, \mathbf{6 s}$ and $\mathbf{6 t}$ in moderate to good yields (up to 51\% over four steps). Even though a 34\% yield for dicarbazole based TAB $6 \mathbf{t}$ seems only moderate at first sight, one has to note that this corresponds to an average yield of $76 \%$ per step over four steps, which is still remarkable. In general, all substituents were well tolerated by the reaction, allowing for moderate to excellent yields, confirming the novel domino reaction as a new powerful approach to highly functionalized aromatic systems.

As reaction mechanism we proposed a linear four-step domino process as depicted in (Fig 3d): the first step consists of a vinylogous Michael addition, resulting in dicyanoolefin 3. As $\mathbf{3}$ possesses two stereocenters, it consequently yields a mixture of two diastereomers, which we isolated and characterized during our investigations for $\mathbf{3 a}$. Compound $\mathbf{3}$ can then again be deprotonated on the nitroalkane unit to allow intramolecular cyclization by attacking one cyano-group to give sixmembered ring containing structure 4 . The resulting imine can now undergo an imine-enamine tautomerization to yield cyclohexadiene derivative $\mathbf{5}$, which we isolated and characterized for $\mathbf{5 a}$. In the final step, the six-ring in $\mathbf{5 a}$ is aromatized towards the desired product $\mathbf{6}$.

\section{Synthesis of hexaarylbenzene}

After having developed the synthetic route towards key precursor $\mathbf{6 a}$, we next aimed at the synthesis of the HAB derivative bearing six different aryl rings. To achieve this goal, we carried out big scale synthesis of starting materials: Nitrostyrene $\mathbf{1 a}$ and $\alpha, \alpha$-dicyanoolefin $\mathbf{2 a}$ were synthesized according to literature known procedures ${ }^{30-32}$ in multi-gram scale (Fig. 4a). To ensure our four-step domino reaction was applicable in gram scale, we performed an upscaling, resulting in $1.64 \mathrm{~g}$ of product in $78 \%$ yield ( $94 \%$ per step). This result again verifies the novel reaction as key stepping stone for HAB synthesis, albeit the reaction time had to be prolonged to 24 hours. Starting with more than $1 \mathrm{~g}$ of $\mathbf{6 a}$, we chose a set of conventional transformations to carry out a straightforward synthesis of selected HAB 15 (Fig. 4c). To keep the whole procedure facile and general, we decided to introduce the fourth aromatic ring as late as possible. Therefore, we choose first to transform 6a with copper(II)bromide and iso-amyl nitrite in a Sandmeyer-type reaction to the corresponding bromine derivative 7, which could be obtained in $98 \%$ yield. Subsequently, we chose the hydrolysis and the Hoffman rearrangement as follow-up steps, in order to convert the nitrile group into the corresponding amine 9. Unfortunately, literature reported procedures were not feasible for bromine 7 as the derivative is quite insoluble in polar or aqueous media ${ }^{33}$. For this reason, we chose to modify the procedure by changing the ratio of DMSO to $\mathrm{H}_{2} \mathrm{O}_{2}$ from 2:1 to 5:1, which resulted in reduced reaction time of 30 minutes with $91 \%$ isolated yield of $\mathbf{8}$. Since reaction 
conditions of both steps of following Hoffmann-rearrangement are quite similar, we combined these two steps in an unprecedented one-pot procedure to give amine 9 in overall yield of $89 \%$.

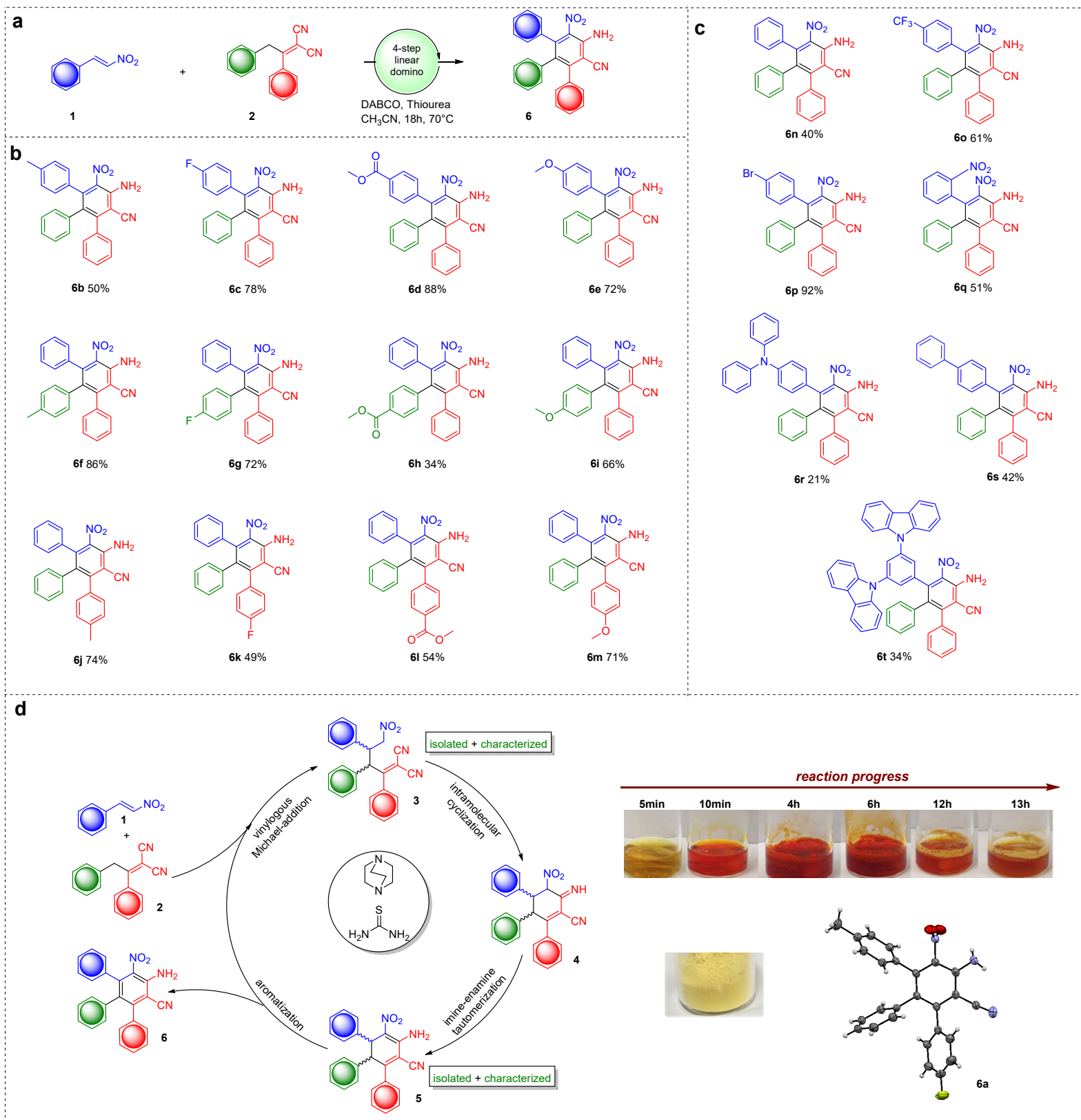

Fig. 3 | Scope of the novel four-step linear domino reaction. a, General reaction equation. b, Systematic evaluation of functional group influence on isolated product yield. c, Scope expansion. d, Reaction mechanism, color change of the reaction mixture over time and crystal structure of $\mathbf{6 a}$. 


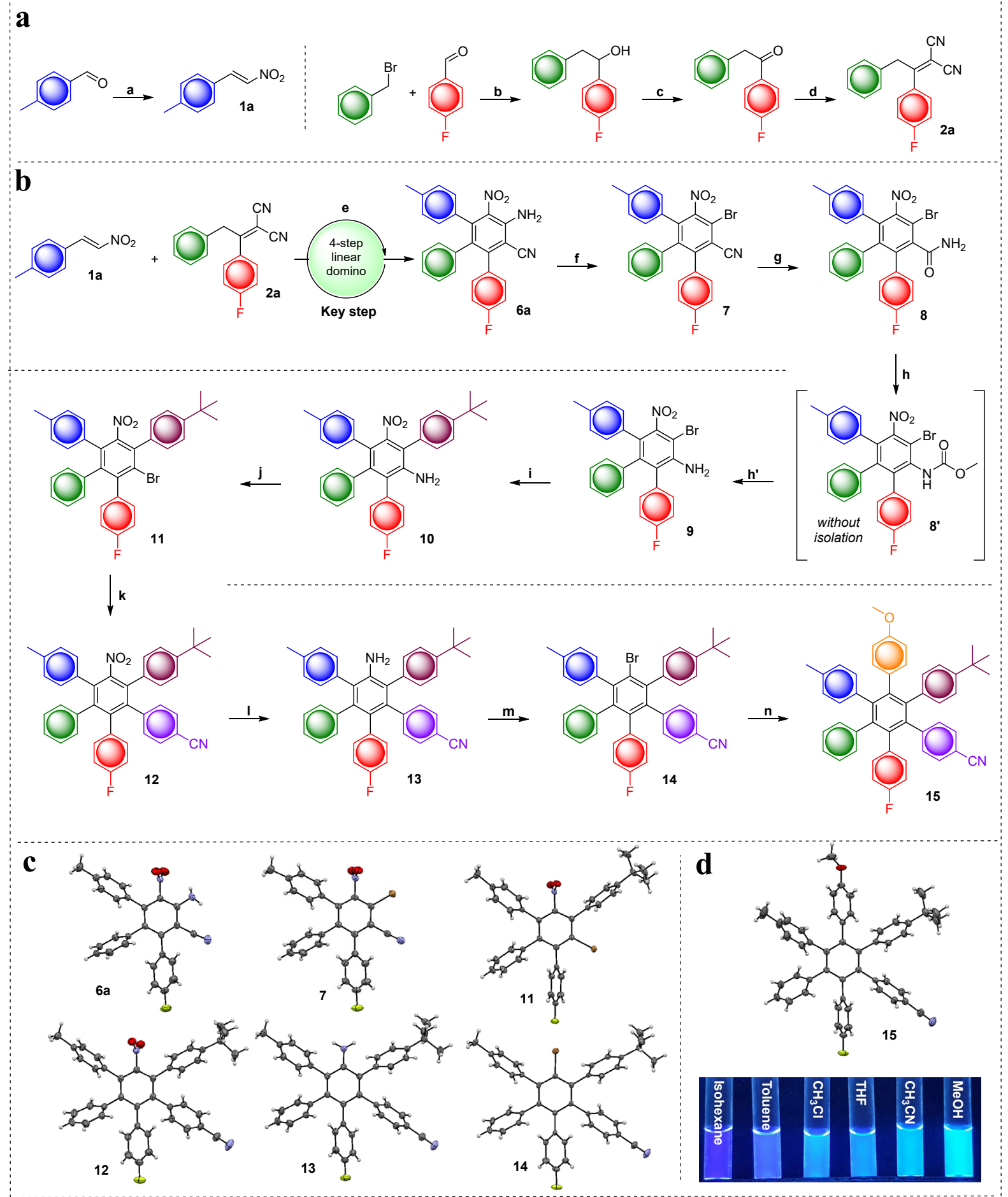

Fig. 4 | Total synthesis of hexaarylbenzene with domino reaction as key step. a, Synthesis of starting materials: a) $\mathrm{CH}_{3} \mathrm{NO}_{2}, \mathrm{NH}_{4} \mathrm{OAc}$, reflux, $95 \%$ yield; b) $1 . \mathrm{Mg}, \mathrm{Et}_{2} \mathrm{O}$, benzylbromide, r.t. $\rightarrow 40{ }^{\circ} \mathrm{C}$, 2 . 4fluorobenzaldehyde, $-78^{\circ} \mathrm{C} \rightarrow 40^{\circ} \mathrm{C}>99 \%$ yield; c) Pyridinium chlorochromate, $\mathrm{CH}_{2} \mathrm{Cl}_{2}$, r.t., $89 \%$ yield; d) malononitrile, $\mathrm{NH}_{4} \mathrm{OAc} / \mathrm{HOAc}$, Toluene, $120^{\circ} \mathrm{C}, 92 \%$ yield. b, e) Large scale synthesis of $6 \mathbf{a}(78 \%$ yield); f) $\mathrm{CuBr}_{2}$, iso-amyl nitrite, $\mathrm{CH}_{3} \mathrm{CN}, 50^{\circ} \mathrm{C}, 98 \%$ yield; g) $\mathrm{H}_{2} \mathrm{O}_{2} / \mathrm{DMSO}$, r.t.; $91 \%$ yield; h) 1 . bis(methoxycarbonyl)phenyliodine, $\mathrm{KOH}, \mathrm{MeOH}, 0^{\circ} \mathrm{C} \rightarrow$ r.t., $2 . \mathrm{KOH} / \mathrm{MeOH}$ (aq. $30 \%$ ), reflux, $89 \%$ 
yield; i) $\mathrm{Pd}$ (dppf) $\mathrm{Cl}_{2} \cdot \mathrm{DCM}, \mathrm{Cs}_{2} \mathrm{CO}_{3}$, corresponding boronic acid, toluene $/ \mathrm{H}_{2} \mathrm{O}, 100^{\circ} \mathrm{C}, 88 \%$ yield; j) $\mathrm{CuBr}_{2}$, iso-amyl nitrite, $\mathrm{CH}_{3} \mathrm{CN}, 50^{\circ} \mathrm{C}, 94 \%$ yield; $\left.\mathrm{k}\right) \mathrm{Pd}(\mathrm{dppf}) \mathrm{Cl}_{2} \cdot \mathrm{DCM}, \mathrm{Cs}_{2} \mathrm{CO}_{3}$, corresponding boronic acid, toluene $/ \mathrm{H}_{2} \mathrm{O}, 100^{\circ} \mathrm{C}, 80 \%$ yield; i) $\mathrm{Zn}, \mathrm{FeCl}_{3}, \mathrm{DMF} / \mathrm{H}_{2} \mathrm{O}$ (5:1), ultrasonic bath, $66 \%$ yield; $\mathrm{m}$ ) isoamyl nitrite, bromoform, $100^{\circ} \mathrm{C}, 59 \%$ yield; n) $\mathrm{Pd}(\mathrm{dppf}) \mathrm{Cl}_{2} \cdot \mathrm{DCM}, \mathrm{Cs}_{2} \mathrm{CO}_{3}$, corresponding boronic acid, toluene $/ \mathrm{H}_{2} \mathrm{O}, 100^{\circ} \mathrm{C}, 57 \%$ yield. Overall yield: $7.5 \%$ (12.4\% starting from domino product $\left.6 \mathbf{6 a}\right)$. c, Crystal structures of $\mathbf{6 a}, 7, \mathbf{1 1}, 12$ and $\mathbf{1 4}$. d, Crystal structure and solvatochromic effect of $\mathbf{1 5}$.

Next, we chose modified Suzuki conditions ${ }^{34}$, employing $\mathrm{Pd}(\mathrm{dppf}) \mathrm{Cl}_{2} \cdot \mathrm{DCM}$ as catalyst, which gave the desired four-ring system 10 in $88 \%$ yield, starting from compound $\mathbf{9}$. The obtained 4tbutylaryl derivative 10 subsequently underwent Sandmeyer type transformation, towards the corresponding bromine 11 in $94 \%$ yield. Attempts to reduce the nitro group in 11 before Suzukicoupling, were not successful, because all reactions employed did either not show any conversion or resulted in dehalogenation. We therefore decided to change the order of steps and introduced the fifth ring through Suzuki-coupling, before reduction of the nitro group, in $80 \%$ yield of $\mathbf{1 2}$. Reduction of nitro-group in 4-cyanoaryl derivative 12 turned out to be quite challenging, since different established methodologies did not result in conversion to the desired amine 13. To our delight, after thorough screening (see Table S2), we found that using combination of $\mathrm{Zn}$ and $\mathrm{FeCl}_{3}$ in $\mathrm{DMF} / \mathrm{H}_{2} \mathrm{O}$ led to success. After converting amine 13 to bromine derivative 14 via modified Sandmeyer type reaction using bromoform/isoamyl nitrite, we performed the final palladium catalyzed reaction towards target $\mathrm{HAB}$ system $\mathbf{1 5}$, with six different aromatic rings around central benzene core.

We succeeded to obtain a single crystal of topologically achiral (due to practically free internal rotation in solution around $\mathrm{C}-\mathrm{C}$ bonds connecting the aryls to benzene core) but propeller chiral $C_{1}$ non-symmetrical 15 and determined its structure (see Fig. S5-S9). The unit cell contains dimers of two enantiomers each of 15, stacked face-to-face in an antiparallel manner (see Fig. S5 and Fig. S6). Within the monoclinic I2/a achiral space group, four of these dimers inhabit the unit cell. The packing motif consists of dimers, which are slightly tilted with respect to each other. Dispersion interactions dominate the packing; for example, the aliphatic methyl and tertiary butyl groups of 15 are clustered together (Fig. S7). Also, nitrile and methoxy substituents pair up in the structure(Fig. S8), whereas the fluorine substituents form quite rare $\mathrm{C}-\mathrm{H} \cdots \mathrm{F}-\mathrm{C}$ hydrogen bonds ${ }^{35}$ to various aromatic protons of neighboring molecules (Fig. S9).

\section{Photophysical study}

With a substantial amount of $\mathbf{1 5}$ in hand, we decided to conduct a basic photochemical study of the compound, taking the solvatochromic effect in observed blue fluorescence (see Fig. 4d) into account. It is important to mention, that HPB itself does not possess fluorescent properties, which gives evidence that the new route of synthesis opens the door for tailormade optical properties of future compounds. To shed light on the optical properties of the electronic ground state of $\mathbf{1 5}$, absorption spectra were measured in cyclohexane exhibiting absorptions in the UV region below $330 \mathrm{~nm}$ with a shoulder-like peak around $285 \mathrm{~nm}$ (see Fig. S10). First insights into excited states properties of $\mathbf{1 5}$ came from steady state and time resolved fluorescence measurements. HAB $\mathbf{1 5}$ exhibits in cyclohexane, THF and acetonitrile strong fluorescence in the UV and blue range of the optical spectrum, with fluorescence maxima and fluorescence quantum yields depending on solvent polarity: $353 \mathrm{~nm}\left(\Phi_{\mathrm{Flu}}=0.15\right)$ in cyclohexane, $380 \mathrm{~nm}\left(\Phi_{\mathrm{Flu}}=0.16\right)$ in $\mathrm{THF}$ and $415 \mathrm{~nm}$ $\left(\Phi_{\text {Flu }}=0.19\right)$ in acetonitrile (see Fig. S11). The trend of increasing fluorescence quantum yield 
with increasing solvent polarity is also reflected in the fluorescence lifetimes, which are prolonged from $2.2 \mathrm{~ns}$ in cyclohexane to $3.0 \mathrm{~ns}$ in THF and $4.8 \mathrm{~ns}$ in acetonitrile (see Fig. S12). Transient absorption spectroscopy based on nanosecond laser flash photolysis exciting 15 at $266 \mathrm{~nm}$ in cyclohexane helped to investigate the $\mathrm{S}_{1}-\mathrm{S}_{\mathrm{N}}$ and $\mathrm{T}_{1}-\mathrm{T}_{\mathrm{N}}$ transitions. The transient absorption of the first singlet excited state was obtained from taking the transient absorption spectrum within the nanosecond laser pulse and exhibited a spectrum dominated by a negative transient absorption signal in the UV and blue region of the optical spectrum which is best explained by the strong fluorescence of $\mathbf{1 5}$ in this spectral region and a featureless transient absorption band in the visible and NIR region with a maximum around $640 \mathrm{~nm}$. Well in line with the obtained fluorescence lifetimes - reflecting the singlet first excited state lifetime - this transient absorption feature decays within the instrument response function of our nanosecond transient absorption setup, and gives rise to a new set of transient absorptions with a dominating maximum around $360 \mathrm{~nm}$ and a smaller shoulder-like maximum around $600 \mathrm{~nm}$ (see Fig. S13 and Fig. S14). This transient absorption decays on the microsecond time scale. The monoexponential fit of the time profile at $360 \mathrm{~nm}$, measured in cyclohexane, gives a lifetime of $2.9 \mu \mathrm{s}$. The most plausible rationale for this transient absorption is the triplet first excited state of $\mathbf{1 5}$, which is further corroborated by the observed for a triplet excited state expected - oxygen sensitivity of this transient absorption (see Fig. S14). These optical properties might be further fine-tuned by deliberately designing certain asymmetrically substituted HABs with explicit push-pull substituents (electron donating and withdrawing groups) in order to allow specific "delayed fluorescence" applications, e.g., in blue OLEDs $^{36,37}$.

\section{Conclusion}

The results presented here demonstrate that the developed versatile high-yielding metal-free fourstep domino reaction can be used as a key step to access specific asymmetrically substituted HABs in high overall yield and without involvement of statistical steps. We believe that this efficient domino reaction, featured by functional group tolerance, modularity, simplicity and scalability, will likely be of great utility for creating diverse and hitherto inaccessible HAB scaffolds for a wide range of applications. Hence, this generally applicable synthetic procedure should give access to all possible 60 position isomers for HABs with six different substituents, as well as to symmetric configurations, when three or more substituents are equal - including the last six constitution isomers, which are not yet reported to the best of our knowledge. Chiral separation of appropriately substituted HABs could also allow to exploit chirality of the chromophore, e.g. to achieve circularly polarized luminescence ${ }^{38}$.

\section{Data and materials availability}

All data supporting the findings of this study are available within the paper or Supplementary Information. Crystallographic data for compounds 6a, 7, 11, 12, 13, 14 and 15 are available free of charge from the Cambridge Crystallographic Data Centre under CCDC identifiers 2055879, 2055878, 2055881, 2055884,
(www.ccdc.cam.ac.uk/structures/).

1 Ames, G. R. The Synthesis of Substituted Terphenyls. Chem. Rev. 58, 895-923, (1958). Catalysts. Top Curr Chem (Cham) 377, 31, (2019). 
3 Taylor, R. D., MacCoss, M. \& Lawson, A. D. Rings in drugs. J. Med. Chem. 57, $5845-$ 5859, (2014).

4 Schaub, T. A., Padberg, K. \& Kivala, M. Bridged triarylboranes, -silanes, -amines, and phosphines as minimalistic heteroatom-containing polycyclic aromatic hydrocarbons: Progress and challenges. J. Phys. Org. Chem. 33, (2019).

5 Chen, S.-H., Jiang, Kai, Xiao, Ying, Cao, Xi-Ying, Arulkumar, Mani, Wang, Zhao-Yang. Recent endeavors on design, synthesis, fluorescence mechanisms and applications of benzazole-based molecular probes toward miscellaneous species. Dyes Pigm. 175, 108157, (2020).

Schramm, S. \& Weiß, D. Fluorescent heterocycles: Recent trends and new developments. 128, 103-179, (2019).

7 König. 14 Organic Dyes in Photocatalytic Reductive C-H Arylations. (2019).

8 Fu, Y. \& Finney, N. S. Small-molecule fluorescent probes and their design. RSC Advances 8, 29051-29061, (2018).

Lambert, C. Hexaarylbenzenes-prospects for toroidal delocalization of charge and energy. Angew. Chem. Int. Ed. Engl. 44, 7337-7339, (2005).

10 Vij, V., Bhalla, V. \& Kumar, M. Hexaarylbenzene: Evolution of Properties and Applications of Multitalented Scaffold. Chem. Rev. 116, 9565-9627, (2016).

11 Wu, J., Pisula, W. \& Müllen, K. Graphenes as potential material for electronics. Chem Rev 107, 718-747, (2007).

12 Narita, A., Wang, X. Y., Feng, X. \& Müllen, K. New advances in nanographene chemistry. Chem Soc Rev 44, 6616-6643, (2015).

13 Englert, J. M., Malig, J., Zamolo, V. A., Hirsch, A. \& Jux, N. HBC-porphyrin--close contact chromophores. Chem. Commun. 49, 4827-4829, (2013).

14 Suginome, S., Sato, H., Hori, A., Mishima, A., Harada, Y., Kusaka, S., Matsuda, R., Pirillo, J., Hijikata, Y., Aida, T. One-Step Synthesis of an Adaptive Nanographene MOF: Adsorbed Gas-Dependent Geometrical Diversity. J. Am. Chem. Soc. 141, 15649-15655, (2019).

15 Umeyama, T., Hanaoka, T., Yamada, H., Namura, Y., Mizuno, S., Ohara, T., Baek, J., Park, J., Takano, Y., Stranius, K., Tkachenko, N. V., Imahori, H. Exclusive occurrence of photoinduced energy transfer and switching of its direction by rectangular pi-extension of nanographenes. Chem. Sci. 10, 6642-6650, (2019).

16 Chen, L., Hernandez, Y., Feng, X. \& Müllen, K. From nanographene and graphene nanoribbons to graphene sheets: chemical synthesis. Angew. Chem. Int. Ed. Engl. 51, 76407654, (2012).

17 Feng, X., Pisula, W. \& Müllen, K. Large polycyclic aromatic hydrocarbons: Synthesis and discotic organization. Pure Appl. Chem. 81, 2203-2224, (2009).

18 Kojima, T. \& Hiraoka, S. Selective alternate derivatization of the hexaphenylbenzene framework through a thermodynamically controlled halogen dance. Org. Lett. 16, 10241027, (2014).

19 Martin, M. M., Dill, M., Langer, J. \& Jux, N. Porphyrin-Hexaphenylbenzene Conjugates via Mixed Cyclotrimerization Reactions. J. Org. Chem. 84, 1489-1499, (2019).

20 Ito, H., Segawa, Y., Murakami, K. \& Itami, K. Polycyclic Arene Synthesis by Annulative pi-Extension. J. Am. Chem. Soc. 141, 3-10, (2019). 
21 Lungerich, D., Reger, D., Holzel, H., Riedel, R., Martin, M. M., Hampel, F., Jux, N. A Strategy towards the Multigram Synthesis of Uncommon Hexaarylbenzenes. Angew. Chem. Int. Ed. Engl. 55, 5602-5605, (2016).

22 Kosaka, T., Inoue, Y. \& Mori, T. Toroidal Interaction and Propeller Chirality of Hexaarylbenzenes. Dynamic Domino Inversion Revealed by Combined Experimental and Theoretical Circular Dichroism Studies. J. Phys. Chem. Lett. 7, 783-788, (2016).

23 Suzuki, S., Segawa, Y., Itami, K. \& Yamaguchi, J. Synthesis and characterization of hexaarylbenzenes with five or six different substituents enabled by programmed synthesis. Nat. Chem. 7, 227-233, (2015).

Rueping, M. \& Atodiresei, I. 6.13 C-C Bond Formation: Cascade or Domino Reaction. Comprehensive Chirality 6, 345-373, (2012).

25 Nicolaou, K. C., Edmonds, D. J. \& Bulger, P. G. Cascade reactions in total synthesis. Angew. Chem. Int. Ed. 45, 7134-7186, (2006).

26 Tietze, L. F. Domino Reaction: Concepts for Efficient Organic Synthesis. (2014).

Held, F. E., Guryev, A. A., Fröhlich, T., Hampel, F., Kahnt, A., Hutterer, C., Steingruber, M., Bahsi, H., von Bojnicic-Kninski, C., Mattes, D. S., Foertsch, T. C., Nesterov-Mueller, A., Marschall, M., Tsogoeva, S. B. Facile access to potent antiviral quinazoline heterocycles with fluorescence properties via merging metal-free domino reactions. Nat.

Commun. 8, 15071, (2017).
Bock, C. M., Parameshwarappa, G., Bonisch, S., Neiss, C., Bauer, W., Hampel, F., Gorling, A., Tsogoeva, S. B. Generation of Complex Azabicycles and Carbobicycles from Two Simple Compounds in a Single Operation through a Metal-Free Six-Step Domino Reaction. Chem. Eu. J. 22, 5189-5197, (2016).

29 Grau, D., Grau, B. W., Hampel, F. \& Tsogoeva, S. B. Three-Component Domino Knoevenagel/Vinylogous Michael Reaction: Entry to Challenging o-Terphenyls. Chem. Eur. J. 24, 6551-6556, (2018).

30 Grau, B. W., Bönisch, Simon, Neuhauser, Alexander, Hampel, Frank, Görling, Andreas, Tsogoeva, Svetlana B. Facile Access to Challenging ortho-Terphenyls via Merging Two Multi-Step Domino Reactions in One-Pot: A Joint Experimental/Theoretical Study. ChemCatChem 11, 3982-3992, (2019).

31 Floresta, G., Cilibrizzi, A., Abbate, V., Spampinato, A., Zagni, C., Rescifina, A. 3D-QSAR assisted identification of FABP4 inhibitors: An effective scaffold hopping analysis/QSAR evaluation. Bioorg. Chem. 84, 276-284, (2019).

32 Xue, D., Li, J., Zhang, Z. T. \& Deng, J. G. Efficient method for the synthesis of polysubstituted benzenes by one-pot tandem reaction of vinyl malononitriles with nitroolefins. J. Org. Chem. 72, 5443-5445, (2007).

33 Zhang, C.-L., Gao, Z.-H., Liang, Z.-Q. \& Ye, S. N-Heterocyclic Carbene-Catalyzed Synthesis of Multi-Substituted Benzenes from Enals and $\alpha$-Cyano- $\beta$-methylenones. $A d v$. Synth. Catal 358, 2862-2866, (2016).

34 Gildner, P. G. \& Colacot, T. J. Reactions of the 21st Century: Two Decades of Innovative Catalyst Design for Palladium-Catalyzed Cross-Couplings. Organometallics 34, 54975508, (2015).

35 Thakur, T. S., Kirchner, M. T., Bläser, D., Boese, R. \& Desiraju, G. R. C-H $\cdots$ F-C hydrogen bonding in 1,2,3,5-tetrafluorobenzene and other fluoroaromatic compounds and the crystal structure of alloxan revisited. CrystEngComm 12, 2079-2085, (2010). 
36 Watanabe, S. \& Kido, J. Hexaphenylbenzene Derivatives for Blue Organic Light-emitting Devices. Chem. Lett. 36, 590-591, (2007).

37 Nakanotani, H., Tsuchiya, Y. \& Adachi, C. Thermally-activated delayed fluorescence for light-emitting devices. Chem. Lett., (2021).

38 Mori, T. Propeller Chirality: Circular Dichroism and Circularly Polarized Luminescence. 151-175, (2020).

Acknowledgments We thank H. Maid, C. Placht (FAU) for assistance with NMR-measurements and M. Dzialach, W. Donaubauer (FAU) for assistance with high-resolution mass spectrometry measurements. S.B.T. thanks Deutsche Forschungsgemeinschaft (DFG) for funding by grants TS87/17-1; TS87/23-1, and the Solar Technologies go Hybrid (Research Network SolTech) initiative of the Bavarian State Ministry for Science, Research and Art for research funding. N.J. thanks the Deutsche Forschungsgemeinschaft (DFG, SFB953) for research grant Nr. 182849149.

Competing interests The authors declare no competing interests. 